\title{
Unravelling basic mechanisms in addiction and neuropsychiatric disorders
}

\author{
Daniela Reich-Erkelenz ${ }^{1} \cdot$ Andrea Schmitt $^{2} \cdot$ Peter Falkai $^{2}$
}

Published online: 12 October 2015

(C) Springer-Verlag Berlin Heidelberg 2015

Since functional imaging studies have shown a significant association between particularly the nucleus accumbens (NAcc), and the pathophysiology of drug addiction, Seifert et al. [1] investigated potential volume difference of the NAcc in heroin-addicted patients compared to healthy controls and additionally correlated subcortical volumes with clinical measurements of negative effects in addiction. Scanning the groups with 3T structural MRI and performing subcortical segmentation analysis plus assessing anxiety and depressive symptoms showed decreased left NAcc volume in heroin-dependent patients. Furthermore, this group's depression score was negatively correlated with the left NAcc volume, while the daily opioid dose was positively correlated with the right amygdala volume. Thus, correlating subcortical structures with negative emotions and opioid doses might be interesting in future studies on heroin dependence. These findings are also reported by Müller et al. [2], who in a postmortem study investigated the NAcc and discuss findings with respect to deep brain stimulation, a technique requiring precise computerassisted planning of implementation. The authors calculated NAcc volumes of heroin addicts compared to healthy controls via morphometry of serial whole-brain sections and found larger total brain volume in the heroin group. NAcc volume, again, was nearly equally decreased in the left and the right hemisphere in the heroin-addicted group.

Andrea Schmitt

Andrea.Schmitt@med.uni-muenchen.de

1 Institute of Psychiatric Phenomics and Genomics, Ludwig-Maximilians-University Munich, Nußbaumstr. 7, 80336 Munich, Germany

2 Department of Psychiatry and Psychotherapy, LudwigMaximilians-University Munich, Nußbaumstr. 7, 80336 Munich, Germany
Further analyses of NAcc volumes in different psychiatric disorders could contribute to improvement of precise targeting and electrode placement using deep brain stimulation. With publication of the DSM-V, gambling disorder meanwhile also has acquired the status as a behavioral addiction. Grant et al. [3] in an MRI study are the first to compare cortical morphometry in untreated subjects with gambling disorder to healthy controls. The authors found significant reductions in cortical thickness in patients, predominantly in right frontal cortical regions. Taking into account the further pronounced right frontal morphometric abnormalities bespeak a neurobiological overlap with substance disorders. Thus, future studies should not only explore the trait versus state nature of these findings but also eventual similarities with other putative behavioral addictions.

In the treatment of bulimia nervosa, the original cognitivebehavioral $(\mathrm{CB})$ maintenance model has widely provided the basis for CBT, but only $40-50 \%$ of treatment completers respond fully and lastingly. Thus, the enhanced CB model (CB-E) extended this approach by encapsulating four additional maintenance factors. Dakanalis et al. [4] evaluated both models allowing for DSM-IV and DSM-5 criteria for bulimic-type eating disorders in a large clinical sample and found the added hypothesized maintenance variables of the CB-E model to improve explanatory utility by better accounting for a greater proportion of variance in dietary restraint and binge eating than the original model. While there exist several studies on self-esteem in anorexia nervosa (AN), so far little was known about AN patient's estimation of other's weight and attractiveness. Horndasch et al. [5] discovered a different perception of other women's bodies belonging to different BMI categories in adolescent AN patients and healthy controls. The observed significant tendency of patient's engagement in physical comparison with others and weight as 
attractiveness ratings might contribute to the maintenance of dysfunctional physical comparison processes.

Studies of patients with the highly heritable attentiondeficit/hyperactivity disorder (ADHD) and ADHD-like traits in healthy subjects strongly bespeak the continuous distribution of ADHD symptoms with impairment of inhibition-related functions as one of the most pronounced cognitive deficits in the general population. Using multiple tests of inhibition-related functions, Polner et al. [6] were the first to investigate the relationship between inhibitory performance and ADHD-like traits in a large sample of healthy adults. Their results show an, albeit small, relationship between individual differences in ADHD-like traits and variance in fundamental inhibition-related functions plus additional effects of negative affect regulation. Further studies on the relationship between genetic factors and the deficit of inhibition-related functions might shed light on the quasi-dimensionality of ADHD.

Experimental evidences point to a neuroprotective effect of the cholinesterase inhibitor donepezil in the treatment of Alzheimer's disease (AD). Talib et al. [7] in their casecontrol study focused on effects of donepezil treatment on reduced phospholipase $\mathrm{A}_{2}$ (PLA2) activity and increased phosphorylation of glycogen synthase kinase 3B (GSK3B), both enzymes being prominently attributed to AD. Patients underwent a 6-month treatment with $10 \mathrm{mg}$ daily dose of donepezil including cognitive assessment and collection of platelet samples before treatment and after 3 and 6 months. While intracellular calcium-independent PLA2 (iPLA2) activity in patients before treatment was lower than in controls, it significantly increased after 3 and 6 months of treatment and was related to cognitive improvement. An additional increase in phosphorylated GSK3B suggests donepezil to delay the progression of $\mathrm{AD}$ in two possible disease mechanisms.

To explore the assumed variability in task design and clinical patient factors responsible for inconsistently reported deficiencies in visuospatial working memory (VSWM) in obsessive-compulsive disorder (OCD), Martoni et al. [8] studied effects of design factors task difficulty, the clinical factors gender, OCD symptom dimension, and duration of illness on VSWM. The authors could confirm a previously reported VSWM deficit in OCD, which noteworthy only was significant for females and thus underlines evidence for sexual dimorphism in this disorder. Significance was found for the impact of more severe OCD symptoms in the symmetry dimension on VSWM, but not for duration of illness. The authors suspect VSWM deficits in OCD to appear more severe with higher task load, possibly mediated through poor task strategy and these mainly in female patients or those with symmetry symptoms of both genders. Rodgers et al. [9] with respect to OCD's heterogeneous phenomenology started an alternative approach via investigating a pure $\mathrm{OCD}$ and a mixed OCD subtype including manifesting obsessions with/without compulsions considering a broad pattern of psychosocial risk factors and comorbid diagnoses. While the pure OCD subtype onset with comorbidities, mainly restricted to phobias, mostly occurred at lower age with few associations with psychosocial risk factors, the mixed OCD subtype was not only higher prevalent but also associated with diverse childhood adversities, more familial burden, and comorbid disorders inclusively those marked by high impulsivity. These results hopefully contribute to improve in-depth insights into OCD-relevant pathophysiological mechanisms. In a letter to the editor, Kohl et al. [10] reflect a recent Science publication on optogenetic stimulation in a mouse model in which repeated stimulation of the orbitofrontal cortexventromedial striatum caused OCD-like symptoms which again could be reduced by fluoxetine and other SSRIs. Since optogenetic stimulation is yet far from being used in humans, the authors advocate deep brain stimulation (DBS) as an alternative neuromodulative procedure which has proven to be useful in the ventral striatum via bringing the network into balance. In this respect, they appeal to combining DBS with behavioral therapy in order to provide a new translational approach to characterize normalization of pathologic network function seen in OCD.

\section{References}

1. Seifert CL, Magon S, Sprenger T, Lang UE, Huber CG, Denier N, Walter M (2014) Reduced volume of the nucleus accumbens in heroin addiction. Eur Arch Psychiatry Clin Neurosci. doi:10.1007/s00406-014-0564-y

2. Müller UJ, Truebner K, Schiltz K, Kuhn J, Mawrin C, Dobrowolny H, Steiner J (2015) Postmortem volumetric analysis of the nucleus accumbens in male heroin addicts: implications for deep brain stimulation. Eur Arch Psychiatry Clin Neurosci. doi:10.1007/s00406-015-0617-x

3. Grant JE, Odlaug BL, Chamberlain SR (2015) Reduced cortical thickness in gambling disorder: a morphometric MRI study. Eur Arch Psychiatry Clin Neurosci. doi:10.1007/s00406-015-0592-2

4. Dakanalis A, Carrà G, Calogero R, Zanetti MA, Gaudio S, Caccialanza R, Clerici M (2014) Testing the cognitive-behavioural maintenance models across DSM-5 bulimic-type eating disorder diagnostic groups: a multi-centre study. Eur Arch Psychiatry Clin Neurosci. doi:10.1007/s00406-014-0560-2

5. Horndasch S, Heinrich H, Kratz O, Mai S, Graap H, Moll GH (2015) Perception and evaluation of women's bodies in adolescents and adults with anorexia nervosa. Eur Arch Psychiatry Clin Neurosci. doi:10.1007/s00406-015-0603-3

6. Polner B, Aichert D, Macare C, Costa A, Ettinger U (2014) Gently restless: association of ADHD-like traits with response inhibition and interference control. Eur Arch Psychiatry Clin Neurosci. doi:10.1007/s00406-014-0531-7

7. Talib LL, Hototian SR, Joaquim HPG, Forlenza OV, Gattaz WF (2015) Increased iPLA2 activity and levels of phosphorylated GSK3B in platelets are associated with donepezil treatment in Alzheimer's disease patients. Eur Arch Psychiatry Clin Neurosci. doi:10.1007/s00406-015-0600-6 
8. Martoni RM, Salgari G, Galimberti E, Cavallini MC, O’Neill J (2015) Effects of gender and executive function on visuospatial working memory in adult obsessive-compulsive disorder. Eur Arch Psychiatry Clin Neurosci. doi:10.1007/s00406-015-0604-2

9. Rodgers S, Ajdacic-Gross V, Kawohl W, Müller M, Rössler W, Hengartner MP, Preisig M (2015) Comparing two basic subtypes in OCD across three large community samples: a pure compulsive versus a mixed obsessive-compulsive subtype. Eur Arch Psychiatry Clin Neurosci. doi:10.1007/s00406-015-0594-0

10. Kohl S, Ebbert L, Sartorius A, Kuhn J (2015) A step forward in elucidating the mystery of OCD. Eur Arch Psychiatry Clin Neurosci. doi:10.1007/s00406-015-0584-2 